\title{
Prognostic importance of lymph node ratio after resection of ampullary carcinomas
}

\author{
Leonardo S. Lino-Silva ${ }^{1}$, Miguel A. Gómez-Álvarez ${ }^{2}$, Rosa A. Salcedo-Hernández ${ }^{2}$, Alejandro E. Padilla- \\ Rosciano $^{2}$, Horacio N. López-Basave ${ }^{2}$ \\ ${ }^{1}$ Surgical Pathology, ${ }^{2}$ Surgical Oncology, Instituto Nacional de Cancerología, Mexico City, Mexico \\ Contributions: (I) Conception and design: All authors; (II) Administrative support: All authors; (III) Provision of study material or patients: All authors; \\ (IV) Collection and assembly of data: All authors; (V) Data analysis and interpretation: LS Lino-Silva, RA Salcedo-Hernández; (VI) Manuscript \\ writing: All authors; (VII) Final approval of manuscript: All authors. \\ Correspondence to: Leonardo S. Lino-Silva, MSc. Avenida San Fernando \# 22, Sección XVI, Tlalpan, Mexico City, México. Email: saul.lino.sil@gmail.com.
}

\begin{abstract}
Background: The prognosis of the lymph node ratio (LNR) in Vater's ampulla carcinomas (VACs) is recently studied. However, there are not enough data in several populations like Latin American people. Our aim is to demonstrate the prognosis significance of the LNR in this setting.

Methods: Pancreaticoduodenectomies for VACs were identified $(n=128)$ from 1980 through 2015. Based on a ROC curve, a cut-off point of 0.1 was assigned for the LNR and the population was divided into two groups for comparison.

Results: The LNR $\geq 0.1$ group was statistically significant associated with recurrence ( $38.5 \%$ s. $19.5 \%)$, pT3-T4 tumors (69.2\% vs. 29.3\%), poorly differentiated tumors (46.2\% vs. $17.5 \%)$, lymphovascular invasion ( 61.5 vs. $17.1 \%)$, perineural invasion $(38.5 \%$ vs. $19.5 \%)$, and positive margins $(15.4 \%$ vs. $2.4 \%)$. In the multivariate analysis, LNR (HR 2.891; CI: 1.987-3.458, P=0.02), LNM (HR 2.945; CI: 2.478-3.245, P=0.002), perineural invasion (HR 3.327; CI: 3.172-4.156, $\mathrm{P}=0.003$ ), and recurrence (HR 3.490; CI: 2.896-4.122, $\mathrm{P}=0.001)$ were associated with lower survival.
\end{abstract}

Conclusions: The LNR is a good predictor of survival and worse oncological outcomes for VACs after resection.

Keywords: Pancreatic cancer; lymph node metastasis; lymph node ratio (LNR)

Submitted May 30, 2018. Accepted for publication Jul 03, 2018.

doi: 10.21037/jgo.2018.07.04

View this article at: http://dx.doi.org/10.21037/jgo.2018.07.04

\section{Introduction}

Surgery remains as the only potential curative treatment for ampullary and periampullary carcinomas [carcinomas of Vater's ampulla or the radial $2 \mathrm{~cm}$ of duodenum surrounding Vater's ampulla, collectively defined as Vater's ampulla carcinomas (VACs)]; however, the 5 -year survival rate has not improved significantly and rarely exceeds $25 \%$ (1). Lymph node metastases (LNMs) have been reported in up to $50 \%$ of patients with resected VACs (2).

In resected specimens, several histopathology factors have been associated with poor prognosis, like tumor stage, size, grade of differentiation, lymph node status, and surgical margins (3). Total number of LNM or total number of lymph nodes examined may be associated with prognosis and an incomplete lymphadenectomy or a bad pathologic processing/examination represents worse prognosis for the patient. This prognosis could be improved if the surgeon performs a good lymphadenectomy or if the pathologist performs a meticulous lymph node identification (4).

Several studies in gastrointestinal cancers had proposed the ratio between the number of LNM and the number of resected lymph nodes [named "lymph node ratio" (LNR), evaluated either as a categorical or as a continuous variable (5)] as a finding associated with decreased overall survival (OS), even suggesting it may be more important 


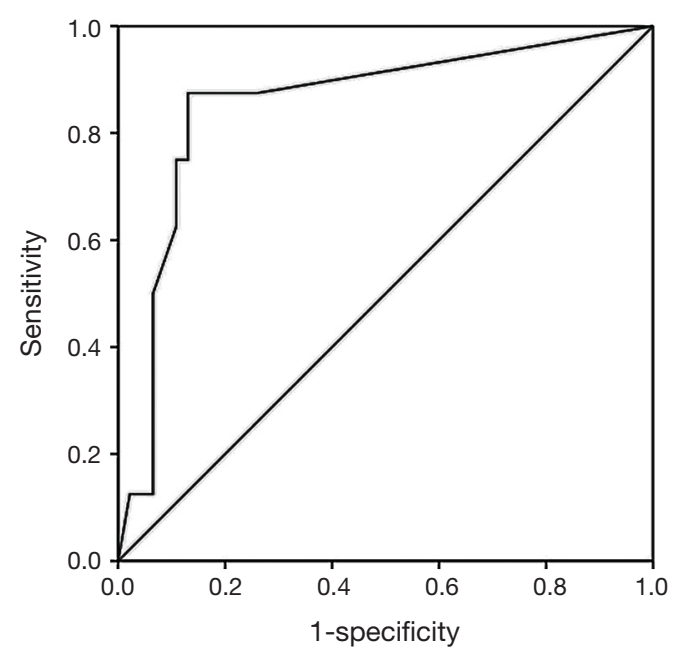

Figure 1 Receiver operator curve (ROC) demonstrating the appropriate cut-off point for the lymph node ratio. It was stated at 0.1 (area under the curve 0.82).

than either the lymph node status or the number of lymph nodes examined (6). These studies show that the LNR was a strong negative prognostic factor In the VAC, the cut-off point has been studied at 0.05 and 0.2 as having prognostic significance for OS, although the range of LNMs is high (42.4-50\%); the average $\mathrm{LN}$ retrieval is around 16 nodes (2,7-9). In our population, LNM in VACs is less frequent and it is not clear if the LNR is also predictive of worse prognoses in a population with a lower LNM rate. Our aim was to characterize the pathological features of VACs in our population in order to investigate the prognostic value of the LNR in patients with pancreaticoduodenectomy for VACs.

\section{Methods}

We performed a cross-sectional study to compare the prognostic significance of the LNR in VACs. We included with curative pancreaticoduodenectomy for VACs at the National Cancer Institute (INCan) in Mexico from January 1980 to December 2015. The data were retrospectively collected and analyzed.

The demographic and clinical information registered included age, sex, symptoms, adjuvant treatment, recurrence, persistence, metastasis, and survival. The pathological details included the tumor size, TNM staging according to American Joint Committee on Cancer, 7th Edition (10), histologic grade, histologic subtype (based on morphology and immunohistochemistry), lymphovascular, perineural, and vascular invasion, surgical margins, lymph node status, and the number of lymph nodes dissected. The LNR was determined by dividing the number of lymph nodes with metastasis by the total of examined lymph nodes. We used a receiver operating characteristic (ROC) curve to determine an appropriate cut-off value for the LNR to predict death (Figure 1), which was between $<0.1$ and $\geq 0.1$, and according with this result, the cases were divided into two groups.

Macroscopic resection was defined as the absence of tumor in the operative field without metastasis (R0). Patients with microscopic positive margins were considered R1 resections and patients with macroscopic evidence of neoplasm in the surgical margins were classified as R2.

The pancreaticoduodenectomy was carried out with or without pylorus preservation according to surgeon preferences. The lymph node dissection in the pancreaticoduodenectomy included the regional lymph nodes to the right-hand side of the celiac and superior mesenteric arteries and all the tissues in the hepatoduodenal ligament, except for the portal vein and hepatic artery and para-aortic dissection. Reconstruction consisted of a pancreaticojejunostomy or a pancreaticogastrostomy. The hepaticojejunostomy was performed $20 \mathrm{~cm}$ distally to the pancreaticojejunostomy, and the duodenojejunostomy was then carried out $50 \mathrm{~cm}$ downstream.

Following surgical resection, if they were candidates to receive chemo radiation, it was administrated by external radiation [total dose of 45 Gy in 25 fractions, with a tumor bed boost of 5.4 Gy in 3 fractions every other day, and 5-fluorouracil (5-FU) or gemcitabine]. The maintenance chemotherapy consisted of $5-\mathrm{FU}\left(375-500 \mathrm{mg} / \mathrm{m}^{2} /\right.$ day $)$ or gemcitabine $\left(1,000 \mathrm{mg} / \mathrm{m}^{2}\right)$.

\section{Statistical analysis}

For numerical data we used mean with standard derivation or a median with range, depending on the distribution, and for categorical variables we used absolute or relative frequencies. The association measures were calculated using Pearson's Chi-square or Fischer's test. Survival was estimated using the Kaplan-Meier method. The evaluation of the independent factors of survival was performed with the Cox regression model controlling for confounders (when the $\mathrm{P}$ value $<0.05$ in the bivariate analysis). The ROC curve was applied to determine the best cut-off point for the LNR. All the statistical analyses were performed using the SPSS software package for Windows 22.0 (SPSS IBM, USA). The 
statistical significance was set at $\mathrm{P}<0.05$.

\section{Results}

From 108 patients, 54 (50\%) were male and 54 were female. The median age was 55.8 years, with a range of 32 to 79 years. A large percentage of the patients (79\%) had jaundice at the diagnosis. An analysis of the intraoperative factors demonstrated a median estimated blood loss of $741 \mathrm{~mL}$ (range, 100-2,000 mL). The median operative time was $7.058 \mathrm{~h}$ with a range of 3 to $10.5 \mathrm{~h}$. Fifty-six $(51.9 \%)$ of the intraoperative blood transfusions required intraoperative packed red blood cells. The $74 \%$ of tumors were of the intestinal type, and $26 \%$ were of the pancreatobiliary type. The predominant $\mathrm{T}$ stage was T2 and T3 with $46 \%$ and $37 \%$, while $\mathrm{T} 1$ was diagnosed in $13 \%$ and $\mathrm{T} 4$ in $1.9 \%$, with $\mathrm{N} 1$ disease in $31.5 \%$ of the cases. Perineural invasion was identified in $24.1 \%$ and lymphovascular invasion in $27.8 \%$. An R0, or microscopically negative resection, was achieved in $94.4 \%$ of the patients. The median number of the lymph nodes sampled was 15 .

The clinicopathologic data between the groups are summarized in Table 1 . The LNR $\geq 0.1$ group was predominant in the males $(69.2 \%$ vs. $43.9 \%)$, which was associated with adverse pathologic factors such as recurrence $(38.5 \%$ vs. $19.5 \%)$, pT3-T4 tumors $(69.2 \%$ vs. $29.3 \%)$, poorly differentiated tumors $(46.2 \%$ vs. $17.5 \%)$, lymphovascular invasion ( $61.5 \%$ vs. $17.1 \%)$, perineural invasion $(38.5 \%$ vs. $19.5 \%)$, and positive surgical margins (15.4\% vs. $2.4 \%)$.

Table 2 describes the results of the survival analysis. The mean follow-up was 55 months (range, 32-79 months) and a total of 16 patients $(56.2 \%)$ died of disease. A higher percentage $(53.8 \%$ vs. $2.4 \%$ ) of the patients in the LNR $\geq 0.1$ group died, while there was a 5 -year OS rate of $97.6 \%$ for the patients in the LNR $<0.1$ group compared with $24 \%$ of the patients in the $\mathrm{LNR} \geq 0.1$ group $(\mathrm{P}<0.001)$ (Figure 2$)$. In the univariate analysis, the clinicopathologic parameters associated with decreased survival were the LNR, the presence of LNM, lymphovascular invasion, perineural invasion, and recurrence. However, in the multivariate analysis, the LNR (HR 2.891; CI: 1.987-3.458, P=0.02), the presence of LNM (HR 2.945; CI: 2.478-3.245, P=0.002), perineural invasion (HR 3.327; CI: 3.172-4.156, $\mathrm{P}=0.003$ ), and recurrence (HR 3.490; CI: 2.896-4.122, $\mathrm{P}=0.001$ ) were associated with decreased survival.

\section{Discussion}

Our data showed that in our population, the LNM was less prevalent $(31.5 \%$ vs. $50 \%$ ), and a LNR $\geq 0.1$ was associated with death $(53.8 \%$ vs. $2.4 \%)$ and was an independent risk factor in multivariate analysis. These data are in accord with previous reports. Hsu et al. (8) evaluated 212 VAC patients who had received radical surgery with a median number of lymph node retrieved of 13 (range, 3-53), a median follow-up of 32.6 months, with a mortality rate of $50 \%$,a median OS was 65.8 and a LNR $>0.056$ associated with poor prognosis in multivariate analysis. Falconi et al. (9) evaluated 90 patients with a median number of 16 examined lymph nodes (range, 5-47); 50\% of the patients had LNM. The 5 -year DSS was $75 \%, 49 \%, 38 \%$, and $0 \%$ for $\mathrm{LNR}=0$, $\mathrm{LNR}>0$ and $\leq 0.2$, LNR $>0.2$, and $\leq 0.4$, and $\mathrm{LNR}>0.4$ ( $\mathrm{P}=0.002)$, respectively. Sakata et al. (2) identified the LNR at a 0.1 cut-off point as an independent predictor for OS in a series with a median of $26 \mathrm{LNs}$ retrieved and a $50 \%$ of patients with positive LNs.

We do not have a satisfactory explanation for the low LNM rate in our cohort because our cases were in a similar proportion with respect to the histologic grade, lymphovascular invasion, perineural invasion, and clinical stage. A plausible explanation is that most of our cases (74\%) were of the intestinal type, and only $26 \%$ were of the pancreatobiliary type.

Finally, the LNR $>0.1$ was associated with recurrence ( $38.5 \%$ vs. $19.5 \%, \mathrm{P}=0.049$ ), poorly differentiated tumors ( $46.2 \%$ vs. $17.5 \%, \mathrm{P}=0.001)$, lymphovascular invasion (61.5\% vs. $17.1 \%, \mathrm{P}=0.01)$, perineural invasion $(38.5 \%$ vs. $19.5 \%, \mathrm{P}=0.049)$ and positive margins $(15.4 \%$ vs. $2.4 \%, \mathrm{P}=0.012)$. In the multivariate analysis, these factors remained associated with decreased survival. The data are in accord with the published data; in particular, LNM has been proposed as a major negative prognosis factor for VACs because it is associated with post-operative liver metastasis and poor OS $(11,12)$. Also, Roland et al. (13) demonstrated that a LNR $\geq 0.15$ was more likely to have T3-T4 tumors, lymphovascular, perineural invasion and to develop recurrent disease.

There are some limitations to our study, like the retrospective nature of the study, the long study period, different surgeons with varying abilities performed the operations for these patients, and the treatment strategies might have changed over time, thus contributing to 
Table 1 Associations between the lymph node ratio and clinicopathological parameters in patients with Vater's ampulla carcinoma

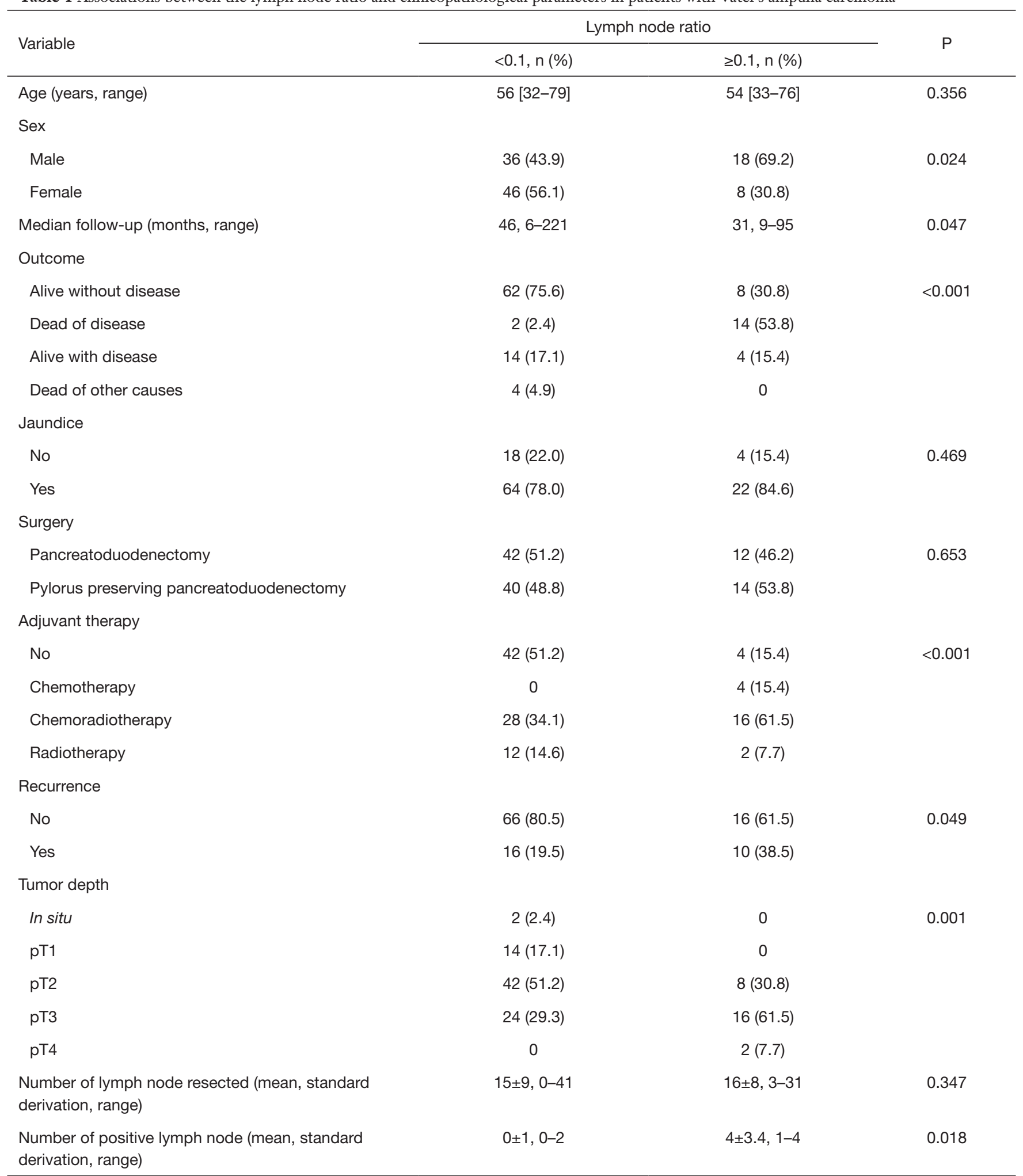

Table 1 (continued) 
Table 1 (continued)

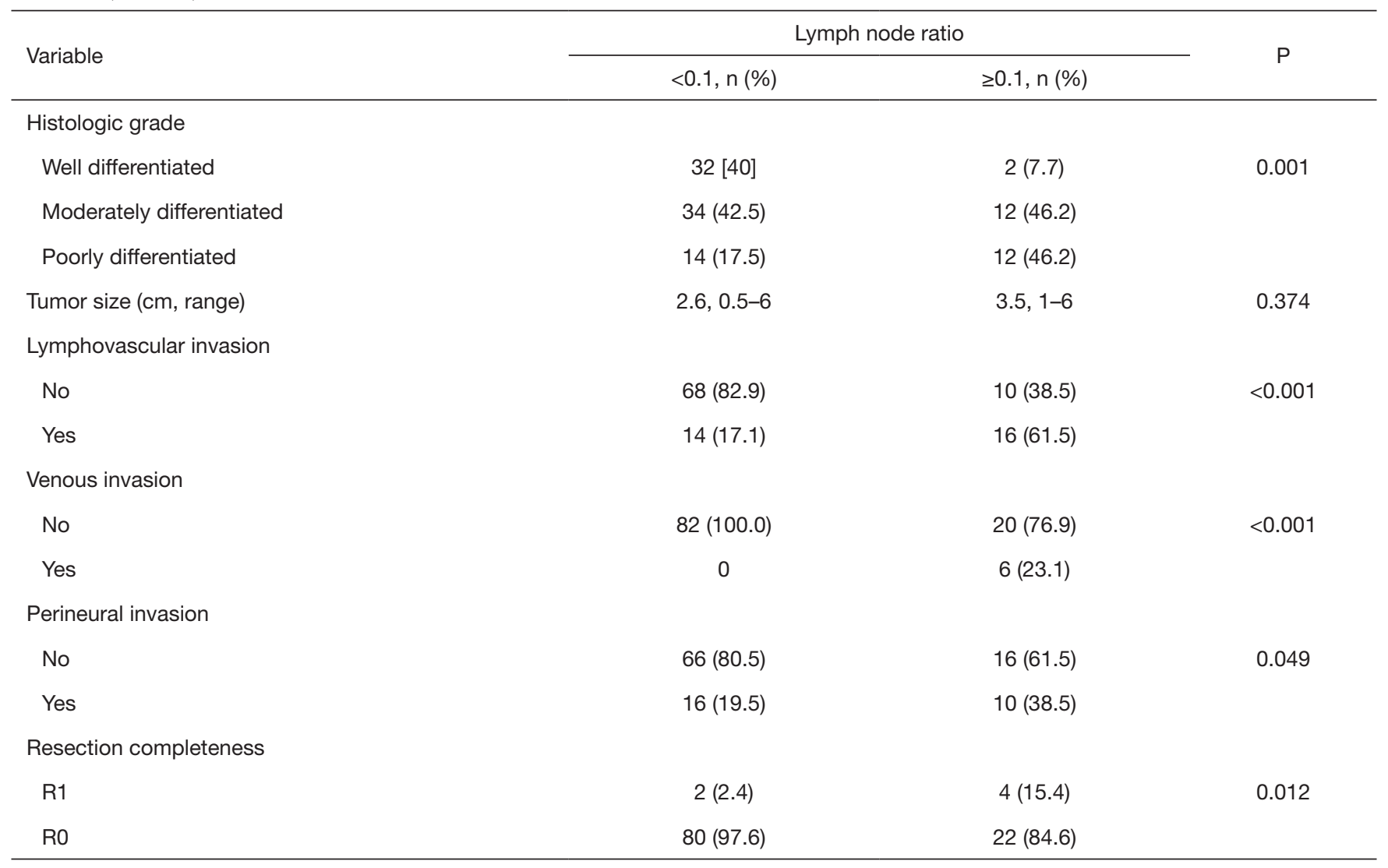

Table 2 Correlations between cancer-specific survival and various clinicopathological variables

\begin{tabular}{|c|c|c|c|c|c|c|}
\hline Variable & \multicolumn{3}{|c|}{ Univariate analysis } & \multicolumn{3}{|c|}{ Multivariate analysis } \\
\hline Lymph node ratio & 4.649 & $3.163-6.135$ & $<0.001$ & 2.891 & $1.987-3.458$ & 0.02 \\
\hline Tumor depth & 3.245 & $0.268-3.987$ & 0.256 & - & - & - \\
\hline Lymph node metastasis & 4.812 & $3.337-6.286$ & $<0.001$ & 2.945 & $2.478-3.245$ & 0.002 \\
\hline Lymphovascular invasion & 2.943 & $1.459-3.269$ & $<0.001$ & 2.968 & $0.575-4.531$ & 0.194 \\
\hline Perineural invasion & 13.482 & $11.543-17.696$ & $<0.001$ & 3.327 & $3.172-4.156$ & 0.003 \\
\hline Recurrence & 3.329 & $2.931-3.547$ & $<0.001$ & 3.490 & $2.896-4.122$ & 0.001 \\
\hline
\end{tabular}

different therapeutic outcomes. Finally, the pathological method of retrieving LNs from specimens has varied over time, with new, more effective protocols and techniques for $\mathrm{LN}$ retrieval during the last 5 years. Further, larger studies are needed to verify our results in other populations.

\section{Conclusions}

In conclusion, the LNR is a strong predictor for longterm survival in patients with pancreaticoduodenectomy for VAC. 


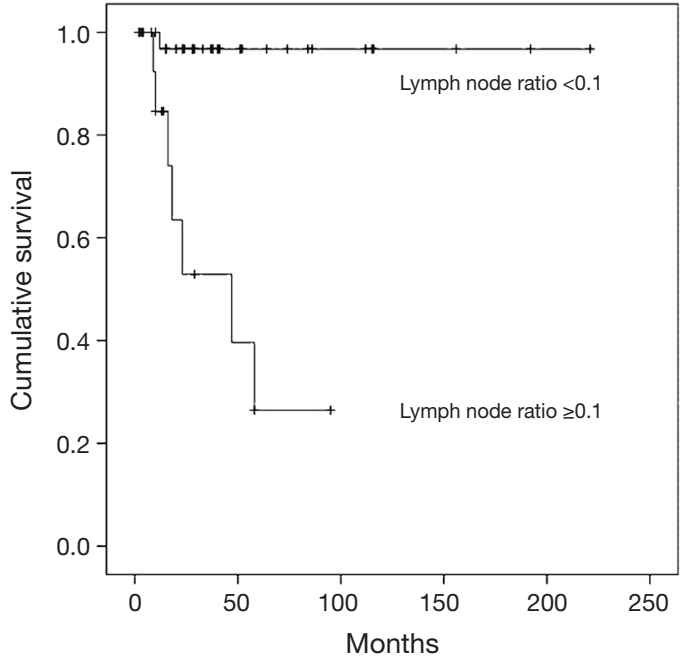

Figure 2 Kaplan Meier curves showing the differences in overall survival according to lymph node ratio $(\mathrm{P}<0.001)$.

\section{Acknowledgements}

This work was awarded as The Best Poster Award in "The 6th Biennal Congress of the Asian-Pacific HepatoPancreato-Biliary-Association"/"The 29th Meeting of Japanese Society of Hepato-Biliary-Pancreatic Surgery" at Yokohama, Japan. June 7-10, 2017.

\section{Footnote}

Conflicts of Interest: The authors have no conflicts of interest to declare.

Ethical Statement: This study was conducted following the statements of Helsinki Declaration and was approved by the Ethics in Investigation Board of National Cancer Institute (Mexico, No. IRB00007348) with a waiver of informed consent, with approval number REV/16/42.

\section{References}

1. Cameron JL, Riall TS, Coleman J, et al. One thousand consecutive pancreaticoduodenectomies. Ann Surg 2006;244:10-5.

2. Sakata J, Shirai Y, Wakai T, et al. Assessment of the nodal status in ampullary carcinoma: the number of positive lymph nodes versus the lymph node ratio. World J Surg 2011;35:2118-24.

3. Brennan MF, Kattan MW, Klimstra D, et al. Prognostic nomogram for patients undergoing resection for adenocarcinoma of the pancreas. Ann Surg 2004;240:293-8.

4. Berger AC, Watson JC, Ross EA, et al. The metastatic/ examined lymph node ratio is an important prognostic factor after pancreaticoduodenectomy for pancreatic adenocarcinoma. Am Surg 2004;70:235-40; discussion 240.

5. Strobel O, Hinz U, Gluth A, et al. Pancreatic adenocarcinoma: number of positive nodes allows to distinguish several N categories. Ann Surg 2015;261:961-9.

6. Pawlik TM, Gleisner AL, Cameron JL, et al. Prognostic relevance of lymph node ratio following pancreaticoduodenectomy for pancreatic cancer. Surgery 2007;141:610-8.

7. Hurtuk MG, Hughes C, Shoup M, et al. Does lymph node ratio impact survival in resected periampullary malignancies? Am J Surg 2009;197:348-52.

8. Hsu CH, Chen TD, Tsai CY, et al. Prognostic Value of the Metastatic Lymph Node Ratio in Patients With Resectable Carcinoma of Ampulla of Vater. Medicine (Baltimore) 2015;94:e1859.

9. Falconi M, Crippa S, Domínguez I, et al. Prognostic relevance of lymph node ratio and number of resected nodes after curative resection of ampulla of Vater carcinoma. Ann Surg Oncol 2008;15:3178-86.

10. Edge SB, Byrd DR, Compton CC. Editors. Amulla of Vater. AJCC Cancer Staging Manual (7th Edn). New York: Springer, 2010:237-42.

11. Sierzega M, Nowak K, Kulig J, et al. Lymph node involvement in ampullary cancer: the importance of the number, ratio, and location of metastatic nodes. J Surg Oncol 2009;100:19-24.

12. Pomianowska E, Westgaard A, Mathisen Ø, et al. Prognostic relevance of number and ratio of metastatic lymph nodes in resected pancreatic, ampullary, and distal bile duct carcinomas. Ann Surg Oncol 2013;20:233-41.

13. Roland CL, Katz MH, Gonzalez GM, et al. A high positive lymph node ratio is associated with distant recurrence after surgical resection of ampullary carcinoma. J Gastrointest Surg 2012;16:2056-63.

Cite this article as: Lino-Silva LS, Gómez-Álvarez MA, Salcedo-Hernández RA, Padilla-Rosciano AE, López-Basave HN. Prognostic importance of lymph node ratio after resection of ampullary carcinomas. J Gastrointest Oncol 2018;9(6):11441149. doi: 10.21037/jgo.2018.07.04 